\title{
Trastuzumab, Pertuzumab, and Docetaxel as the First Line for HER-2-Positive Metastatic Breast Cancer among Arabs
}

\author{
Kausar Suleman ${ }^{a, b}$ Ali Hassan Mushtaq ${ }^{c}$ Emaan Haque Ahmed Ali Badran $^{\mathrm{a}, \mathrm{d}}$ \\ Dahish Ajarim $^{a} \quad$ Ahmed Mohammed Elashwah ${ }^{a, e} \quad$ Ahmed Mostafa Gad ${ }^{a}$ d \\ Amgad Shaheen Abdelsamad ${ }^{a, f} \quad$ Ayman Omar ${ }^{a, b, g}$ Khurshid Ahmed Khan ${ }^{a}$ \\ Taher Al-Tweigeri $^{\mathrm{a}} \quad$ Ayman Elshentenawy $^{\mathrm{a}}$ e Adher Alsayed $^{\mathrm{a}}$ \\ a Oncology Center, King Faisal Specialist Hospital and Research Center, Riyadh, Saudi Arabia; ${ }^{b}$ Associate Professor \\ for Al Faisal University, Riyadh, Saudi Arabia; 'Al Faisal University, Riyadh, Saudi Arabia, Riyadh, Saudi Arabia;

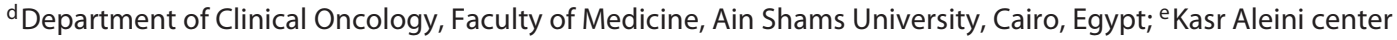 \\ of Clinical Oncology and Nuclear Medicine (NEMROCK), Cairo University, Cairo, Egypt; ${ }^{f}$ Department of Medical \\ Oncology, National Cancer Institute, Cairo University, Cairo, Egypt; ${ }^{9}$ Department of Clinical Oncology and Nuclear \\ Medicine, Suez Canal University Hospital, Ismailia, Egypt
}

\section{Keywords \\ Pertuzumab · Metastatic breast cancer (MBC) • \\ HER-2-positive MBC · Dual targeted therapy}

\begin{abstract}
Introduction: Human epidermal growth factor receptor 2 (HER-2) targeted therapy regimens can improve tumor response in HER-2-positive metastatic breast cancer (MBC), with overall survival benefits. Objective: We evaluated the efficacy of dual HER-2 blockade combined with chemotherapy for HER-2-positive MBC patients as a first-line therapy in our patient population. Patients and Methods: We identified 75 patients at King Faisal Specialist Hospital and Research Center that received trastuzumab, pertuzumab, and docetaxel as a first-line therapy in HER-2 positive MBC in 2013-2016. Results: Median age at diagnosis was 45 years; $54.7 \%$ were estrogen receptor (ER)-positive. $10 \%$ of patients presented with only bone metastasis. The median follow-up time was 36 months with an objective response rate of $74.7 \%$ (complete response [CR] 18.7\%; partial response [PR] 56\%). The 5 -year progression-free survival (PFS) and overall survival (OS) were $21 \%$ and $71.9 \%$ respectively, with a median PFS
\end{abstract}

of 36 months (95\% confidence interval [CI] 23.6-48.4). The 5 -year OS for ER-negative and ER-positive patients was $93.9 \%$ and $59.4 \%$ respectively $(p=0.189) ; 23$ patients experienced grade $1 / 2$ toxicity and 2 patients had grade $3 / 4$ toxicity. In terms of OS and PFS, the site of metastasis did not make any significant difference. Conclusions: First line pertuzumab, trastuzumab, and docetaxel for HER-2-positive MBC patients was found to be an effective and safe therapy in the Saudi population. This finding was consistent with the results seen in the CLEOPATRA trials.

(c) 2020 S. Karger AG, Basel

\section{Introduction}

Anti-human epidermal growth factor receptor 2 (HER-2) directed therapy is established as a standard of care for patients with HER-2-positive breast cancer. Dual HER-2 blockade with pertuzumab used in addition to trastuzumab has been shown to improve progression-

The abstract was presented at the 16th St. Gallen International Breast Cancer Conference, Vienna, Austria on 22-23rd March 2019. 
free survival (PFS) by 6.1 months, as well as increase overall survival (OS) by 15.7 months, with tolerable toxicity in patients in the West [1-3]. This efficacy has also been reported in the neoadjuvant setting [4].

Since these international studies were not conducted on our ethnic group in Saudi Arabia, we aimed to evaluate the safety and efficacy of trastuzumab, pertuzumab, and docetaxel in our cohort of patients as a first-line therapy in the metastatic setting of HER-2-positive patients, and to then compare our results with international data in terms of toxicity and survival outcome.

\section{Methods}

This was a retrospective, observational study carried out in a single institute, which is one of the largest tertiary care centers in the Middle East. Patients who received trastuzumab, pertuzumab, and docetaxel as first-line therapy for de novo metastatic or relapsed HER-2-positive breast cancer were retrospectively identified for the period between May 2013 and September 2016. Pertuzumab was approved as a formulary medication in our institute at the time this study was conducted, and our hospital guidelines adjusted and updated accordingly.

\section{Inclusion Criteria}

- An age of $\geq 18$ years.

- A left ventricular ejection fraction (LVEF) $\geq 50 \%$ as assessed by echocardiography or a multigated acquisition (MUGA) scan.

- A normal complete blood count and differentials (CBCD), and renal and hepatic profiles; all were assessed for response and toxicities.

- HER-2 positivity confirmed by immunohistochemistry (IHC) and/or fluorescence in situ hybridization (FISH) analysis.

\section{Data Collection}

Data were extracted from the electronic health records database. We reviewed each medical record, looking at demographics (age, gender, and comorbidities at presentation); presenting symptoms relating to the tumor; and, in the case of relapse, the type of previous therapy received in the neoadjuvant setting (including trastuzumab-, hormone-, and taxane-based therapy). Complete lab evaluation including $\mathrm{CBCD}$, renal and hepatic profiles, and CA15.3 was done. To determine the basal cardiac function, baseline LVEF was estimated using echocardiography or a MUGA scan, which was done routinely to determine any cardiac toxicity that occurred from the use of the 2 monoclonal antibodies. We also looked at therapy details, the agents used in each line of therapy, the response, and the overall duration of treatment. If patients stopped any line of therapy, then the reason for the change, if any, was also recorded and included toxicity, e.g., neutropenia, allergic reactions, etc. Tumor characteristics such as hormone receptor status and the type of metastasis (visceral, non-visceral, or both) were also noted.

All the tumors were assessed every 3 months as per standard clinical guidelines for response to therapy using the appropriate imaging modality (bone scan or computed tomography [CT]) to assess the response in terms of progression or improvement. Lastly, the date of the last follow-up visit and disease status at this visit were recorded. Data were collected from the period 2013-2016, i.e., for a total of 4 years. Patient and tumor characteristics can be found in Table 1.
Table 1. Patient and clinical characteristics

\begin{tabular}{|c|c|c|}
\hline & $\begin{array}{l}\text { CLEOPATRA } \\
n=402\end{array}$ & $\begin{array}{l}\text { Our study } \\
n=75\end{array}$ \\
\hline \multicolumn{3}{|l|}{ Age, years } \\
\hline Median & 54 & 45 \\
\hline Range & $22-82$ & $27-98$ \\
\hline \multicolumn{3}{|l|}{ Baseline ECHO } \\
\hline$>55 \%$ & n.a. & $58(77.3 \%)$ \\
\hline $50-55 \%$ & n.a. & $17(22.7 \%)$ \\
\hline \multicolumn{3}{|l|}{ Presentation } \\
\hline De novo & $218(54.2 \%)$ & $54(72 \%)$ \\
\hline Relapse & $184(45.8 \%)$ & $21(28 \%)$ \\
\hline \multicolumn{3}{|c|}{ Hormone receptor ${ }^{\mathrm{a}}$ status } \\
\hline Positive & $189(47 \%)$ & $41(54.7 \%)$ \\
\hline Negative & $212(53 \%)$ & $34(45.3 \%)$ \\
\hline \multicolumn{3}{|c|}{ Disease type at screening } \\
\hline Visceral & $314(78 \%)$ & $44(58.7 \%)$ \\
\hline Non-visceral & $88(22 \%)$ & $8(10.7 \%)^{b}$ \\
\hline Both & n.a. & $23(30.7 \%)$ \\
\hline \multicolumn{3}{|c|}{ Concordant metastasis } \\
\hline Yes & n.a. & $20(26.7 \%)$ \\
\hline No & n.a. & $15(20 \%)$ \\
\hline Not available & n.a. & $40(53.3 \%)$ \\
\hline \multicolumn{3}{|c|}{ CNS metastasis (brain) } \\
\hline Yes & n.a. & $5(6.7 \%)$ \\
\hline No & $402(100 \%)$ & $70(93.3 \%)$ \\
\hline \multicolumn{3}{|c|}{ Prior (neo)adjuvant therapy } \\
\hline Yes & $184(46 \%)$ & $21(28 \%)$ \\
\hline No & $218(54 \%)$ & $54(72 \%)$ \\
\hline \multicolumn{3}{|c|}{ (Neo)adjuvant therapy type } \\
\hline Trastuzumab & $47(12 \%)$ & $21(28 \%)$ \\
\hline Anthracycline & $150(37 \%)$ & $21(28 \%)$ \\
\hline Taxane & $91(23 \%)$ & $21(28 \%)$ \\
\hline Hormone & $106(26 \%)$ & $8(11 \%)$ \\
\hline
\end{tabular}

ECHO, echocardiography; CNS, central nervous system; n.a., not available.

${ }^{a}$ Estrogen receptor or progesterone receptor.

b Patients with bone-only metastasis.

\section{Procedures}

Trastuzumab and pertuzumab were given in the first cycle at a dose of $840 \mathrm{mg}+8 \mathrm{mg} / \mathrm{kg}$, and in the subsequent cycles at $420 \mathrm{mg}$ $+6 \mathrm{mg} / \mathrm{kg}$, with no later dose reduction. Docetaxel was started at $75 \mathrm{mg} / \mathrm{m}^{2}$ every 21 days, also with no subsequent dose reduction; it was given for 6-8 cycles, stopped, and then followed by maintenance pertuzumab and trastuzumab as well as hormone treatment for the hormone-positive subgroup according to our local guidelines. All drugs were given intravenously. Pertuzumab was only stopped in the case of disease progression or intolerable side effects. Assessment of the response to treatment was done by radiological imaging, usually a CT/bone scan for bone metastasis if clinically indicated, according to the Response Evaluation Criteria in Solid Tumors (RECIST) v1.1 [5]. Estrogen receptor (ER), progesterone receptor, and HER-2 were determined on the pretreatment biopsy by IHC. HER- 2 was considered positive if $\geq 10 \%$ of tumor cells stained for ER and/or progesterone receptor. HER-2 status was determined by IHC and confirmed with FISH if it was equivocal ( $2+$ ) by IHC; a FISH ratio of $>2$ was considered positive [6]. 


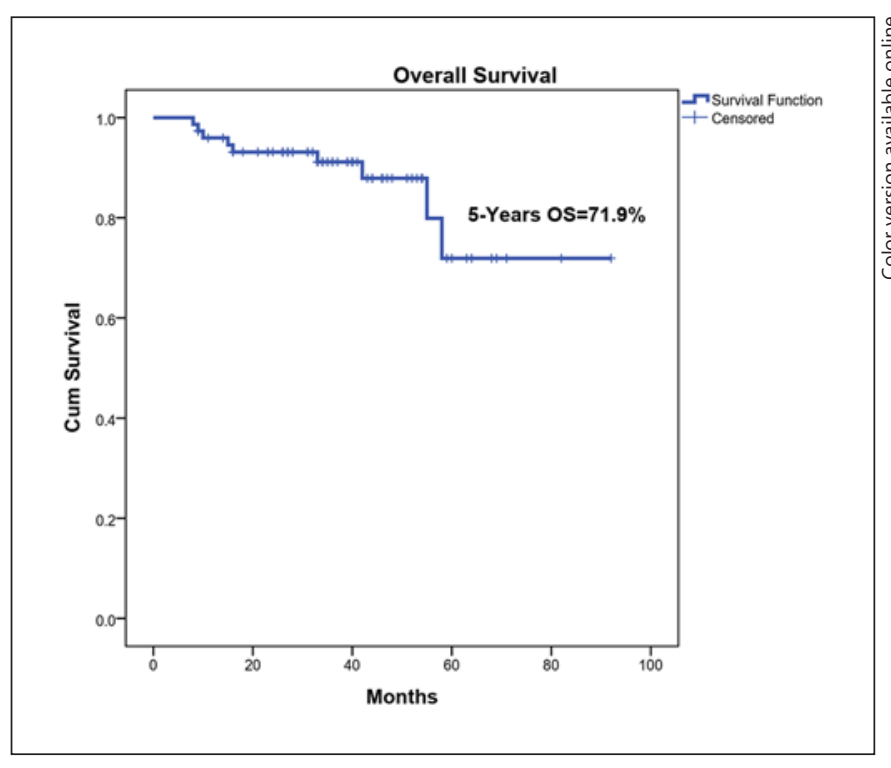

Fig. 1. Kaplan Meier graph for overall survival. 1, positive receptor status; 2 , negative receptor status; Cum, cumulative.

\section{Statistical Methods}

The $\chi^{2}$ test was used to compare the categorical variables expressed as $n(\%)$. Medians were used for the assessment of continuous data as in the case of PFS. OS was defined as the time taken from the initiation of therapy to the death of the patient. PFS was defined as the time from the initiation of therapy to the first evidence of progression assessed by radiological or clinical means. Both OS and PFS were calculated for the overall population and the Kaplan-Meier method was used to approximate the distribution of OS and PFS in the subgroup analysis for hormone receptorpositive and hormone receptor-negative disease as well as for relapse versus de novo disease. Log-rank (Mantel-Cox) test was performed to analyze a difference, if any, with the use of trastuzumab, pertuzumab, and docetaxel for patients with positive versus negative hormone receptor disease, site of metastasis, or relapse versus de novo metastatic disease, all in relation to PFS or OS. $p \leq 0.05$ was considered statistically significant. Response to treatment was defined using RECIST v1.1 [5]. All calculations were performed using SPSS software v20.

\section{Results}

We identified 75 patients that met the inclusion criteria. Median age at diagnosis was 45 years (27-98). The median follow-up time was 36 months (range 8-92). All patients received 6-8 cycles of docetaxel with HER-2 targeted therapies, and then continued dual targeted maintenance therapy. The median number of maintenance cycles of dual targeted therapy was 21 . Of the 75 patients evaluated, $14(18.7 \%)$ showed a complete response (CR) and $42(56 \%)$ a partial response (PR), $8(10.7 \%)$ had stable disease (SD) and 11 (14.7\%) progression of disease (PD). The objective response rate was $74.7 \%$. The 5 -year OS was found to be $71.9 \%$ with a median OS not yet reached

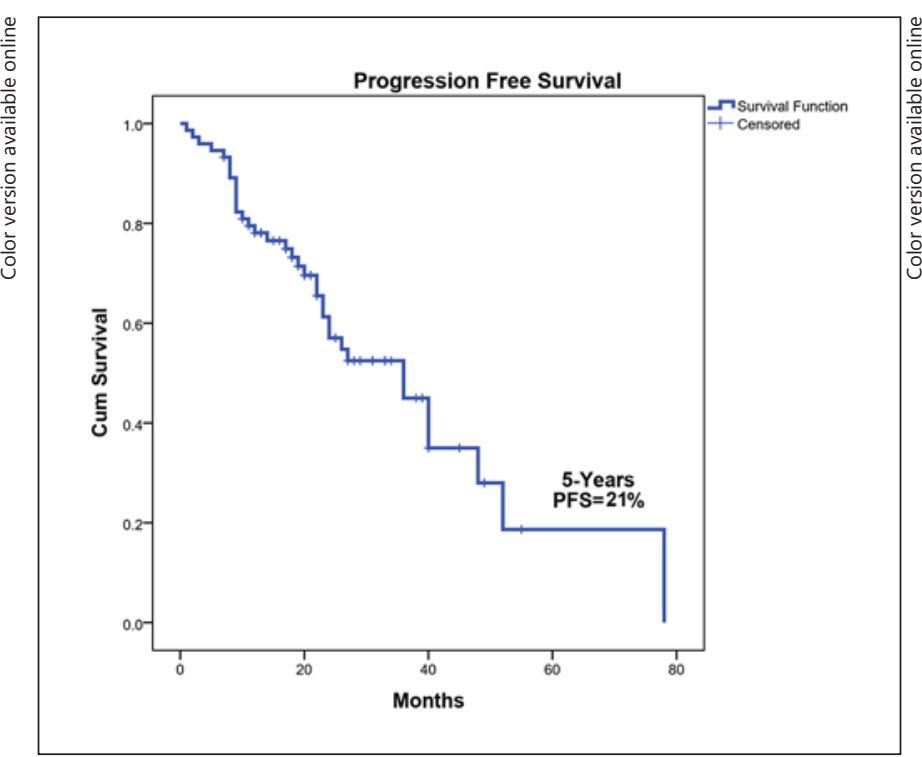

Fig. 2. Kaplan Meier graph for progression-free survival. 1, positive receptor status; 2 , negative receptor status; Cum, cumulative.

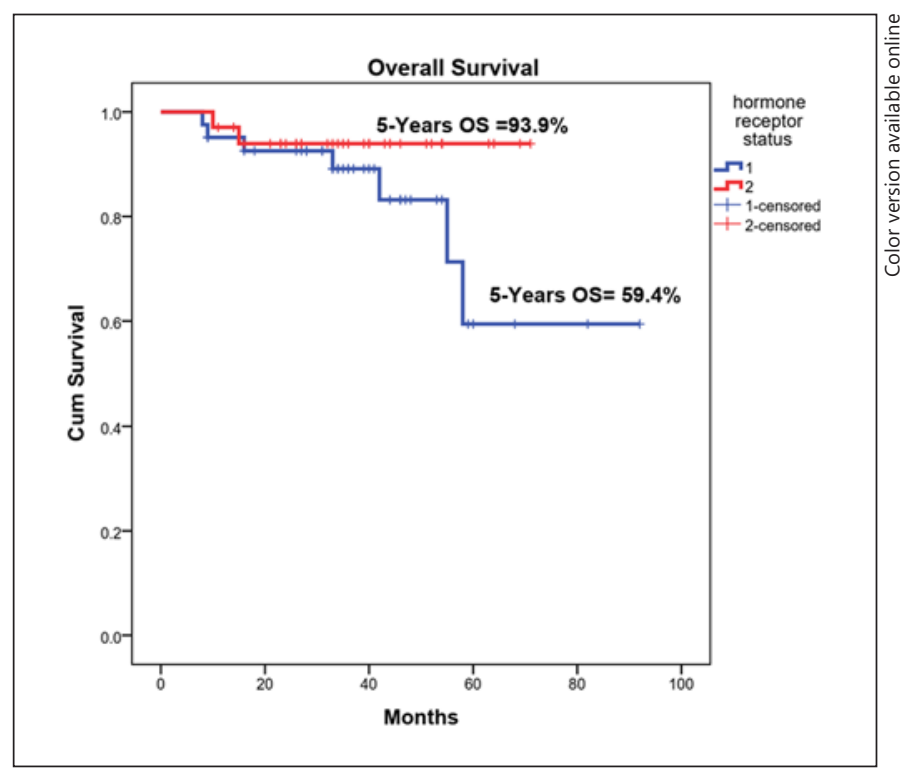

Fig. 3. Kaplan Meier graph for overall survival based on hormone receptor status. 1, positive receptor status; 2 , negative receptor status; Cum, cumulative.

(Fig. 1). The 5-year PFS was found to be $21 \%$ with a median PFS of 36 months (95\% confidence interval [CI] 23.6-48.4) (Fig. 2).

In terms of tumor biology, 41 patients $(54.7 \%)$ had triple-positive hormone receptor status and $34(45.3 \%)$ had $\mathrm{ER} /$ progesterone receptor-negative status. The 5-year OS for hormone receptor-negative patients was $93.9 \%$; for hormone receptor-positive patients, it was $59.4 \%$ ( $p=$ 0.189 ) (Fig. 3). Hormone receptor-negative patients had 


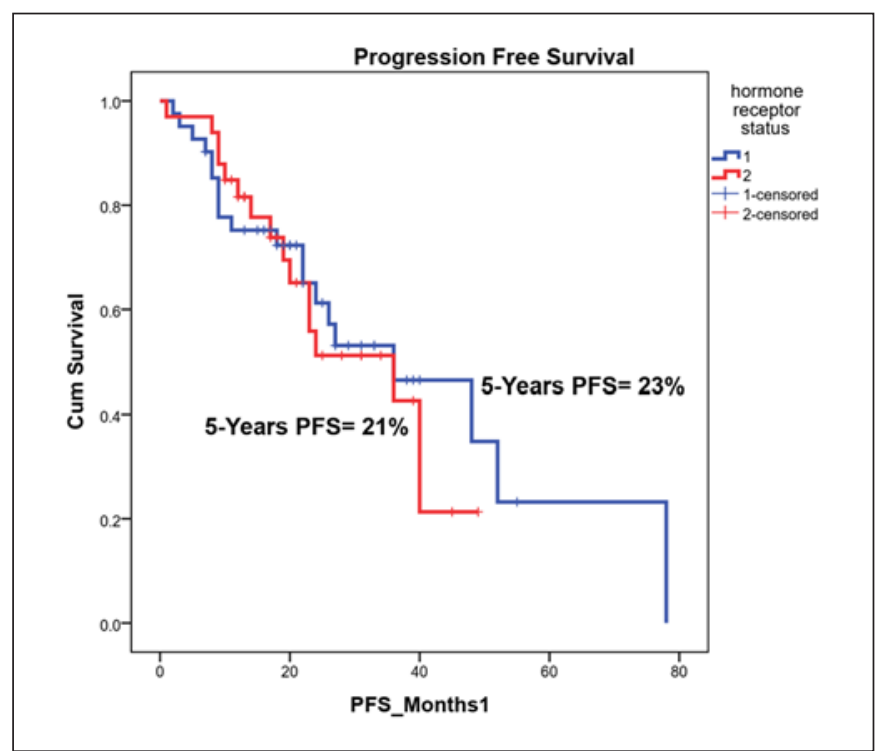

Fig. 4. Kaplan Meier graph for progression-free survival based on hormone receptor status. 1 , positive receptor status; 2 , negative receptor status; Cum, cumulative.

Table 2. Toxicity profile

\begin{tabular}{lc}
\hline Toxicity & $n(\%)$ \\
\hline Hypertension & $3(4 \%)$ \\
Allergic reaction & $5(6.66 \%)$ \\
A drop in ejection fraction & $12(16 \%)$ \\
Thrombocytopenia/neutropenia & $14(18.6 \%)$ \\
Diarrhea & $1(2.3 \%)$ \\
\hline
\end{tabular}

a 5-year PFS of $21 \%$ and hormone receptor-positive patients a 5 -year PFS of $23 \%(p=0.683)$ (Fig. 4$)$.

Forty-four patients $(58.7 \%)$ had visceral metastases, 8 (10.7\%) had non-visceral (bone only) metastasis, and 23 (30.7\%) had both. An analysis was performed to compare the survival outcomes of patients with either visceral or non-visceral metastases, or both. OS and PFS were statistically insignificant at $p=0.723$ and $p=0.592$, respectively. Forty-five patients (60\%) managed to receive secondline therapy upon progression, while $14(18.6 \%)$ received a third line and more treatment regimens. There were 9 patients with primary refractory disease.

Twenty-three patients experienced grade 1-2 toxicity. There were 2 cases of grade 3-4 toxicity, one due to an allergic reaction and the other due to a drop in LVEF; treatment was stopped for both these patients. Refer to Table 2 for a detailed toxicity profile.

Twenty-one patients (28\%) had disease relapse after a prior diagnosis of non-metastatic breast cancer (nonMBC) while 54 patients (72\%) had de novo MBC. For a comparison between de novo and relapse patients' sur- vival outcomes, OS and PFS were calculated. Median PFS was 37 months for de novo patients (95\% CI 14.7-59.3 months) and 36 months for relapse patients (95\% CI 21.4-50.6 months). Log-rank (Mantel-Cox) testing performed in both groups showed $p=0.487$ for PFS and $p=$ 0.317 for OS, indicating no statistically significant differences in survival in either group.

\section{Discussion}

Patients with HER-2-positive MBC are now showing a better prognosis with improved PFS and OS than patients with HER-2-negative disease due to HER-2 targeted therapies $[7,8]$. The phase III CLEOPATRA trial with a median follow-up of 50 months showed that the addition of pertuzumab to trastuzumab and docetaxel significantly improved OS and PFS among HER-2-positive MBC patients. There was an added value of 15.7 months for OS (i.e., 56.5 vs. 40.8 months) and 6.3 months for PFS (18.7 vs. 12.5 months).

Our intention was to prove the efficacy of this combination protocol in our ethnic group, i.e., the Arab population $(n=75)$. The 5 -year OS of our patients was $71.9 \%$ with the median OS not yet reached as $>50 \%$ of the patients were still alive at the end of the study period. However, when comparing our 5-year OS $(71.9 \%)$ with the CLEOPATRA trial's 4-year OS (57.6\%), it was observed that our patients showed a better response. Furthermore, in our study, the median PFS was found to be 36 months, which is better than the 18.7 months demonstrated in the CLEOPATRA trial with 50 months of follow-up. Our data, along with the data of 2 other retrospective studies, show that the results of the CLEOPATRA trial can be replicated with similar, if not better, efficacy of this treatment regimen in a real-life setting $[9,10]$.

The difference in survival outcomes with those of the CLEOPATRA trials may be due to our patient population being younger, with a median age of 45 years (vs. 54 years in the CLEOPATRA trials). These younger patients probably had fewer comorbidities than the relatively older patient population in the CLEOPATRA trial. On the other hand, it has also been observed that breast cancer in younger women ( $<40$ years) presents with a higher grade, i.e., triple-negative or HER-2-positive tumors, and these women also tend to present later, all adding to a poorer survival $[11,12]$.

The ECOG status of the patients started on this firstline therapy was $0-1$. Furthermore, $72 \%$ of our patients were diagnosed with de novo disease and were consequently less prone to resistance. Lastly, the presence of brain metastases was one of the exclusion criteria of the CLEOPATRA trial. Five of our patients $(6.7 \%)$ had brain metastases at baseline; 2 had PR, 2 had SD, and 1 had PD. 
The use of pertuzumab for brain metastatic patients is thus questionable and needs further research. However, these differences did not seem to affect the efficacy profile of this therapy. In fact, our results confirm the usefulness of this dual targeted therapy as a first-line therapy in this group of patients, albeit a small sample.

The objective response rate of $74.7 \%$ in our study is almost comparable to other studies, such as $79 \%$ for the docetaxel group in the PERUSE study [13]. For the paclitaxel group, the objective response rate was higher (83\%), but with reported unfavorable side effects like peripheral neuropathy. Our group also suffered from neutropenia (18.6\%), i.e., higher than the $11 \%$ in the docetaxel group in the PERUSE study. Paclitaxel, the taxane component of this combination therapy, is thus a valid option only in selected patient populations.

In this study, fewer side effects were reported, but this is due to the retrospective nature. Nevertheless, no unexpected or serious toxicity occurred except in 2 patients that were reported to have experienced grade 3/4 toxicity, one due to allergy and the other due to a drop in LVEF. In the CLEOPATRA trials, the intervention group did not have an increase in rates of symptomatic or asymptomatic cardiac dysfunction [1-3]. In our study, the reported cardiac toxicity was higher at $16 \%$ (vs. $3.8 \%$ in the CLEOPATRA trials). One explanation for this may be alternating between echocardiography and a MUGA scan, with the latter being more sensitive as it measures the LVEF exactly without an estimation. Furthermore, in our study, even a trivial drop in LVEF was considered a cardiac toxicity. It is also relevant to note that the Arab population has a higher prevalence of both cardiovascular and coronary artery diseases, which could explain the lower threshold for cardiotoxicity from treatment $[14,15]$.

It has been observed in other studies that median survival is dependent on MBC subtype, with the shortest OS, i.e., 27 months, for ER-negative/HER-2-negative tumors, in comparison to 52 months for ER-negative/HER-2-positive tumors, 76 months for ER-positive/HER-2-negative/Ki67 ${ }^{\text {high }}$ tumors, and 79 months for ER-positive/ HER-2-negative/Ki67 $7^{\text {low }}$ tumors [16].

Our results showed that, in the hormone receptornegative subgroup, the 5-year OS was found to be $93.9 \%$; in the hormone receptor-positive subgroup, it was $59.4 \%$. Although not statistically significant, this trend indicates that ER-negative patients had a better OS in comparison to ER-positive patients. This is an unusual trend that was seen in our patients as it has previously been observed that ER-positive/HER-2 positive (88.7\%) MBC patients had a longer OS than those with ER-negative/HER-2 positive (78.8\%) MBC [17]. Presumably, factors such as small sample size, comorbidities, age, tumor size and degree of metastasis also play an important role in determining the prognosis.

Trastuzumab, Pertuzumab, and Docetaxel in MBC in Saudi Patients
De novo patients, being treatment-naïve, are generally thought to perform better than relapse patients who have previously received some form of neoadjuvant therapy. In our study, the neoadjuvant therapy differed from patient to patient as per the protocol at the time, with 8 patients receiving hormone therapy and all of them having previously received neoadjuvant trastuzumab $(n=21)$. After the diagnosis of relapse of disease, they were started on first-line therapy for metastatic disease with trastuzumab, pertuzumab, and docetaxel. However, our analysis showed no differences in OS or PFS between the de novo and relapse patients. One retrospective study showed that in patients with HER2-positive MBC without prior treatment with trastuzumab had better clinical outcomes than those with prior treatment [18].

Our study suggests that patients receiving this regimen had a remarkable improvement in outcome (PFS and OS). The good clinical result obtained with the combination of trastuzumab and pertuzumab is likely due to the more comprehensive blockade with each monoclonal antibody targeting a distinctive receptor epitope. Pertuzumab binds to HER-2, but, unlike trastuzumab, it binds to the HER-2 subdomain II and thus prevents its dimerization. Due to the similar mechanisms of actions of the 2 drugs, they achieve a much more effective therapy than trastuzumab only; they inhibit receptor dimerization and suppress signaling, ultimately inhibiting the intracellular signaling cascade that promotes cell growth [19]. The effect of using pertuzumab alone was observed in the BO17929 study which showed that using trastuzumab along with pertuzumab resulted in better outcomes [20].

Updated results from the analysis of the CLEOPATRA trial that had a median follow-up of 99 months showed that with an addition of 4 years the OS hazard ratio was 0.69 (95\% CI 0.58-0.82), thus favoring the intervention group. Median OS was 57.1 months in the intervention group and 40.8 months in the control group, i.e., a difference of 16.3 months. The 8-year OS rates were 37 and $23 \%$, respectively. The overall safety profile remained consistent with the previous reports [21].

\section{Strengths and Limitations}

It should be kept in mind that our sample size was fairly small and so it is important to be cautious when interpreting our results. In addition, pertuzumab was used alongside trastuzumab and docetaxel and a 3-drug combination can add a great deal of complexity to the interpretation of the findings. Data on the toxic side effects was incomplete due to the retrospective design of the study. Lastly, the results seen are from a single institute from a retrospective cohort view and not from a randomized prospectively designed study and are thus susceptible to 
bias and error. Valid comparisons can therefore not be made about the efficacy of pertuzumab with a control group. Further research with a larger population and a longer follow-up is warranted.

The strengths of this study should not be forgotten. Firstly, this paper outlines the outcomes of pertuzumabbased therapy for MBC in HER-2-positive patients in a real-life setting. With the current rapidly changing environment in medicine and new treatments being introduced just as swiftly as the updates in best practice, it is necessary to coherently reproduce their value in daily practice. Moreover, this retrospective study looked at long-term outcomes in the Saudi population, a population not included in the CLEOPATRA clinical trials, thus allowing our results to be more generalized to the Arab population despite the small sample size. Furthermore, this study showed that even though most of the patients had locally advanced heavy metastatic disease, they still had a generally good 5-year OS and PFS.

\section{Conclusion}

Our findings suggest that the efficacy seen with the addition of pertuzumab to trastuzumab and docetaxel for HER-2-positive MBC are consistent with the results of the CLEOPATRA trials. These findings in our patient population support the benefit of using this treatment regime in patients with HER-2-positive MBC in Saudi Arabia, but further study is warranted for more conclusive results.

\section{Statement of Ethics}

This research project was conducted in accordance with the Declaration of Helsinki (2000), Good Clinical Practice Guidelines, and the policies and guidelines of the Research Advisory Council of the King Faisal Specialist Hospital and Research Center. It was approved by the medical ethics committee. The identity of the patients studied remained anonymous since no identifying data or protected health information were recorded. All data were password-secured to ensure confidentiality. Consent for publication was granted by the Office of Research Affairs according to institutional internal regulations. The Research Advisory Council has the right of access to the supporting records.

\section{Disclosure Statement}

All authors declare that they have no competing interests.

\section{Funding Sources}

This research received no specific grant from any funding agency in the public, commercial, or not-for-profit sectors.

\section{Author Contributions}

K.S.: study concept and design and manuscript editing. A.H.M.: data acquisition and manuscript writing and editing. E.H.: manuscript writing and data analysis. A.A.B.: study concept and design, statistical analysis, and manuscript editing. D.A.: study design and data analysis and interpretation. A.M.E.: statistical analysis and data interpretation. T.A.-T.: study design and data acquisition, analysis, and interpretation. A.M.G., A.O., A.S.A., K.A.K., A.E., and A.A.: data acquisition, analysis, and interpretation.

\section{References}

1 Swain SM, Baselga J, Kim SB, Ro J, Semiglazov V, Campone M, et al.; CLEOPATRA Study Group. Pertuzumab, trastuzumab, and docetaxel in HER2-positive metastatic breast cancer. N Engl J Med. 2015 Feb;372(8):724-34.

2 Baselga J, Cortés J, Kim SB. Im S-A, Hegg R, Im Y-H, et al. Pertuzumab plus trastuzumab plus docetaxel for metastatic breast cancer. $\mathrm{N}$ Engl J Med. 2012;366(2):109-19.

3 Swain SM, Kim SB, Cortés J, Ro J, Semiglazov $\mathrm{V}$, Campone M, et al. Pertuzumab, trastuzumab, and docetaxel for HER2-positive metastatic breast cancer (CLEOPATRA study): overall survival results from a randomised, double-blind, placebo-controlled, phase 3 study. Lancet Oncol. 2013 May; 14(6): 461-71.

4 Alsayed A, Badran A, Elashwah A, Gad A, Shahin A, Omar A, et al. [Internet] Efficacy, Safety Profiles and Outcomes of Dual Anti Her2 Blockade in Combination with Neoadjuvant Chemotherapy in Locally Advanced (Stage II-III) Her2 Expressing Breast Cancer Patients, Middle East Experience. Pan Arab J Oncol. 2018;Suppl 2(November 2018). Available from: www.amaac.org
5 Nishino M, Jagannathan JP, Ramaiya NH, Van den Abbeele AD. Revised RECIST guideline version 1.1: what oncologists want to know and what radiologists need to know. AJR Am J Roentgenol. 2010 Aug;195(2):2819.

6 Carlson RW, Moench SJ, Hammond ME, Perez EA, Burstein HJ, Allred DC, et al.; NCCN HER2 Testing in Breast Cancer Task Force. HER2 testing in breast cancer: NCCN Task Force report and recommendations. J Natl Compr Canc Netw. 2006 Jul;4 Suppl 3:S1-22.

7 Slamon DJ, Leyland-Jones B, Shak S, Fuchs H, Paton V, Bajamonde A, et al. Use of chemotherapy plus a monoclonal antibody against HER2 for metastatic breast cancer that overexpresses HER2. N Engl J Med. 2001 Mar; 344(11):783-92.

8 Marty M, Cognetti F, Maraninchi D, Snyder R, Mauriac L, Tubiana-Hulin M, et al. Randomized phase II trial of the efficacy and safety of trastuzumab combined with docetaxel in patients with human epidermal growth factor receptor 2-positive metastatic breast cancer administered as first-line treatment: the
M77001 study group. J Clin Oncol. 2005 Jul; 23(19):4265-74.

9 Robert NJ, Goertz HP, Chopra P, Jiao X, Yoo B, Patt D, et al. HER2-Positive Metastatic Breast Cancer Patients Receiving Pertuzumab in a Community Oncology Practice Setting: Treatment Patterns and Outcomes. Drugs Real World Outcomes. 2017 Mar;4(1): $1-7$.

10 De Placido S, Giuliano M, Schettini F, Von Arx C, Buono G, Riccardi F, et al. Human epidermal growth factor receptor 2 dual blockade with trastuzumab and pertuzumab in real life: italian clinical practice versus the CLEOPATRA trial results. Breast. 2018 Apr; 38:86-91.

11 Assi HA, Khoury KE, Dbouk H, Khalil LE, Mouhieddine TH, El Saghir NS. Epidemiology and prognosis of breast cancer in young women. J Thorac Dis. 2013 Jun;Suppl 1:S28.

12 Han W, Kim SW, Park IA, Kang D, Kim SW, Youn YK, et al. Young age: an independent risk factor for disease-free survival in women with operable breast cancer. BMC Cancer. 2004 Nov;4(1):82. 
13 Bachelot T, Ciruelos E, Schneeweiss A, Puglisi F, Peretz-Yablonski T, Bondarenko I, et al. Preliminary safety and efficacy of first-line pertuzumab combined with trastuzumab and taxane therapy for HER2-positive locally recurrent or metastatic breast cancer (PERUSE). Ann Oncol. 2019;30(5):766-73.

14 Al-Nozha MM, Arafah MR, Al-Mazrou YY, Al-Maatouq MA, Khan NB, Khalil MZ, et al. Coronary artery disease in Saudi Arabia. Saudi Med J. 2004 Sep;25(9):1165-71.

15 Noncommunicable diseases country profiles 2011. WHO Global Report. Geneva: WHO; 2011. pp 1-209.

16 Savci-Heijink CD, Halfwerk H, Hooijer GK, Horlings HM, Wesseling J, van de Vijver MJ. Retrospective analysis of metastatic behaviour of breast cancer subtypes. Breast Cancer Res Treat. 2015 Apr;150(3):547-57.
17 Onitilo AA, Engel JM, Greenlee RT, Mukesh BN. Breast cancer subtypes based on ER/PR and Her2 expression: comparison of clinicopathologic features and survival. Clin Med Res. 2009 Jun;7(1-2):4-13.

18 Murthy RK, Varma A, Mishra P, Hess KR, Young E, Murray JL, et al. Effect of adjuvant/ neoadjuvant trastuzumab on clinical outcomes in patients with HER2-positive metastatic breast cancer. Cancer. 2014 Jul;120(13): 1932-8.

19 Baselga J, Swain SM. Novel anticancer targets: revisiting ERBB2 and discovering ERBB3. Nat Rev Cancer. 2009 Jul;9(7):463-75.
20 Cortés J, Fumoleau P, Bianchi GV, Petrella TM, Gelmon K, Pivot X, et al. Pertuzumab monotherapy after trastuzumab-based treatment and subsequent reintroduction of trastuzumab: activity and tolerability in patients with advanced human epidermal growth factor receptor 2-positive breast cancer. J Clin Oncol. 2012 May;30(14):1594-600

21 Swain SM, Miles D, Kim SB. Im Y-H, Im S-A Semiglazov V, et al. End-of-study analysis from the phase III, randomized, doubleblind, placebo (Pla)-controlled CLEOPATRA study of first-line (1L) pertuzumab (P), trastuzumab $(\mathrm{H})$, and docetaxel (D) in patients (pts) with HER2-positive metastatic breast cancer (MBC). J Clin Oncol. 2019 May; 37:1020. 